\title{
IL-1R blockade is not effective in patients with hematological malignancies and severe SARS-CoV-2 infection
}

\author{
Carolina Villegas $^{1}$ (D) - María Poza ${ }^{1}$ - Paloma Talayero ${ }^{2} \cdot$ José Manuel Caro Teller $^{3}$ - Denis Zafra ${ }^{1} \cdot$ Cristina Garcia $^{1}$. \\ Elena Vera ${ }^{1}$. Marta Hidalgo ${ }^{1}$ • Nieves Lopez ${ }^{1}$. Clara Cuellar $^{1}$ • Irene Zamanillo ${ }^{1}$ • Rodrigo Íñiguez ${ }^{1}$ - Estela Paz-Artal ${ }^{2}$. \\ José M. Aguado ${ }^{1,4} \cdot$ J. Martinez-Lopez ${ }^{1}$
}

Received: 15 June 2020 / Accepted: 18 June 2020 / Published online: 25 June 2020

(C) Springer-Verlag GmbH Germany, part of Springer Nature 2020

Keywords Hematological malignancies $\cdot$ IL-1R blockade $\cdot$ SARS-CoV-2

Coronavirus disease 2019 (COVID-19) has been confirmed in more than three million people worldwide. This infection can become serious in $20 \%$ of cases, with a mortality rate of 3$7 \%$, much higher than that of influenza $(1 \%)[1,2]$. Hematological patients are a vulnerable subpopulation due to altered immunity secondary to underlying disease and treatment. Clinical outcomes may be completely different from that of the general population.

Some predictors of a fatal outcome in COVID-19 are age, presence of underlying diseases or secondary infections, and elevated inflammatory markers. This exaggerated systemic immune response, also called cytokine release syndrome (CRS), and as its most severe form macrophage activation syndrome (MAS), could explain the respiratory failure from acute respiratory distress syndrome (ARDS) that is the main cause of mortality in COVID-19[3, 5].

The molecular mechanism underlying this immune system dysregulation includes defects in cytolytic activity, leading to

\section{Key points}

- We report 5 hematological patients diagnosed with SARS-CoV-2 infection (COVID-19) treated with anakinra without any clinical benefit. - IL1R blockade did not show any variation in the tendency for high inflammatory markers in hematological patients with COVID-19.

Electronic supplementary material The online version of this article (https://doi.org/10.1007/s00277-020-04160-w) contains supplementary material, which is available to authorized users.

J. Martinez-Lopez

jmarti01@med.ucm.es

1 Hematology Department Hospital 12 de Octubre, i+12, Universidad Complutense, CNIO, CIBERONC, Madrid, Spain

2 Immunology Department, Hospital Universitario "12 de Octubre," Instituto de Investigación Sanitaria Hospital "12 de Octubre" (imas12), Universidad Complutense, Madrid, Spain a pro-inflammatory cytokine cascade that results in high levels of IL-1, IL-6, IL-18, soluble interleukin 2 receptor (sIL-2R), tumor necrosis factor (TNF), and interferon $\gamma 4$. [6].

Anakinra is an IL-1 receptor antagonist (IL1Ra) with a wide therapeutic range and high safety profile that plays an important role in regulating systemic inflammatory responses $[4,7,8]$. Anakinra may be viewed as an emerging and effective therapy for patients with features of MAS secondary to COVID-19.

We present our experience with anakinra in a subgroup of five patients with hematological diseases and severe SARSCoV-2 infection with a state of hyperinflammation refractory to tocilizumab (TCZ).

During the COVID-19 pandemic, at the Hospital Universitario 12 de Octubre, between 9 Mar 2020 and 9 Apr 2020, 48 patients with hematological malignancies were diagnosed with COVID-19. Thirty-six patients were admitted to the hospital because they presented with radiological
3 Pharmacy Department Hospital Universitario 12 de Octubre, Instituto de Investigación Sanitaria Hospital "12 de Octubre" (imas12), Universidad Complutense, Madrid, Spain

4 Unit of Infectious Diseases, Hospital Universitario "12 de Octubre," Instituto de Investigación Sanitaria Hospital "12 de Octubre" (imas12), Universidad Complutense, Madrid, Spain 


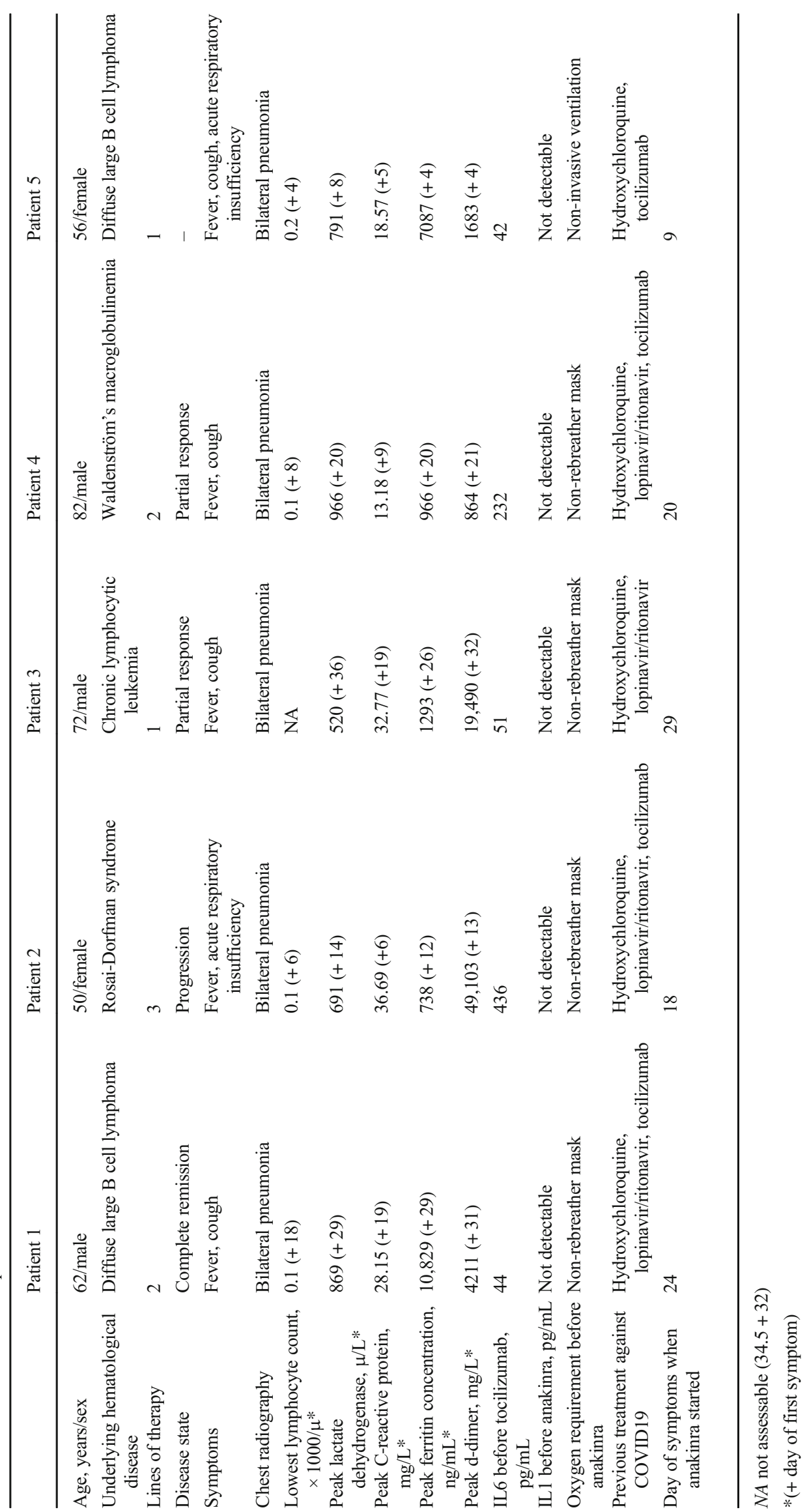




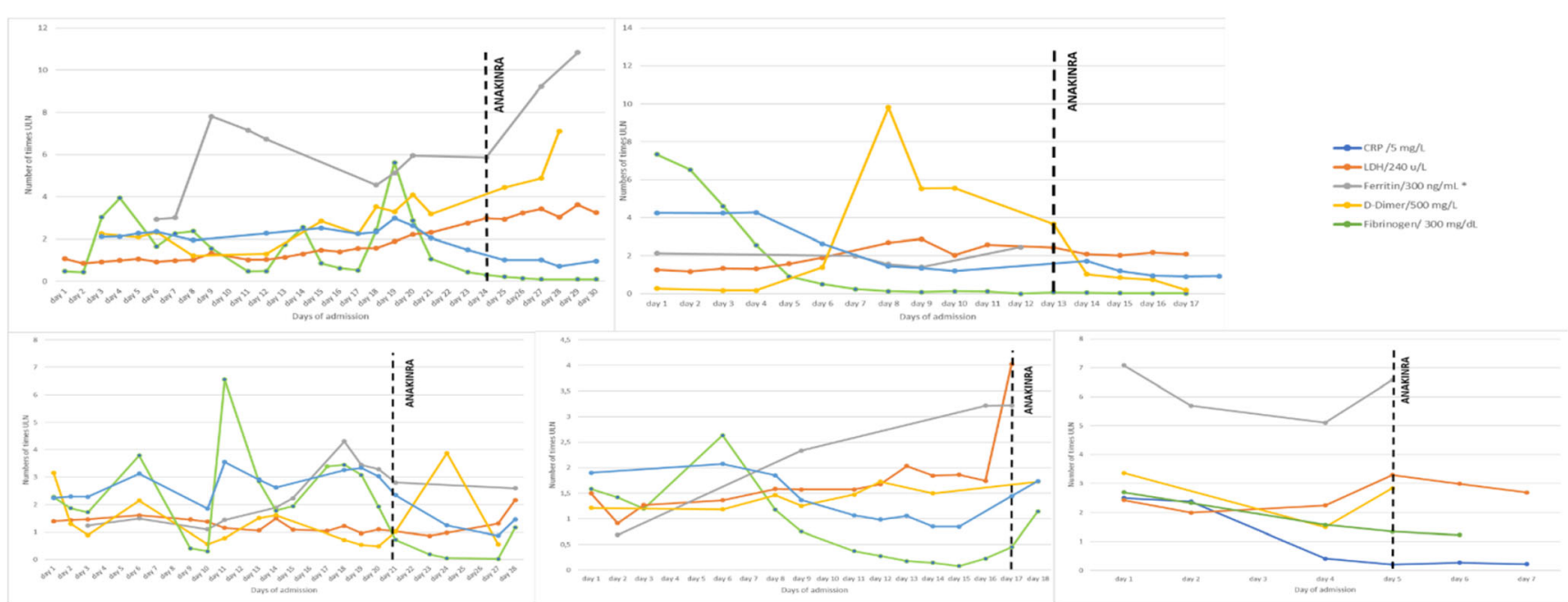

Fig. 1 Timeline of inflammatory markers C-reactive protein, lactate dehydrogenase, ferritin, d-dimer, and fibrinogen

abnormalities or required supplemental oxygen. The initial treatment was established to be hydroxychloroquine (HCQ, $200 \mathrm{mg}$ bid) plus lopinavir/ritonavir (LPV/RTV, 200/50 mg bid). Patients with high initial inflammatory markers or without response were treated with corticosteroids and TCZ (400 or $600 \mathrm{mg}$ intravenous, adjusted by weight). If no clinical improvement occurred, the patients were treated with anakinra (100 mg bid day $1,100 \mathrm{mg} / 24 \mathrm{~h} 2-5$ days, subcutaneous).

Five patients diagnosed with severe SARS-CoV-2 infection by RT-PCR were treated with anakinra at our center (Table 1). The median age was 62 years (range 50-82 years), and the hematological diagnoses were diffuse large B cell lymphoma $(n=2)$, chronic lymphocytic leukemia $(n=1)$, Waldeström's macroglobulinemia $(n=1)$, and RosaiDorfman syndrome $(n=1)$. All patients were undergoing active treatment of first to third-line therapy, and the hematological malignancy status was complete remission $(n=1)$, partial remission $(n=2)$, progression $(n=1)$, and pending evaluation $(n=1)$. Patients were admitted at a median 4 days (range 18 days) after initial symptom onset. The most common initial symptoms were fever $(n=5)$, cough $(n=4)$, and shortness of breath $(n=2)$. Chest X-ray showed bilateral pneumonia, and chest computed tomography (CT) demonstrated diffuse ground glass opacities. All of the patients required high-flow supplemental oxygen $\left(\mathrm{FiO}_{2} \geq 40 \%\right)$ or non-invasive mechanical ventilation. The clinical and analytical characteristics of each patient are given in Table 1. Treatment was started with HCQ $(n=5)$, LPV/RTV $(n=4)$, and corticosteroids $(n=5)$, without clinical improvement. All patients were also given a single-dose of intravenous TCZ between days 5 and 25 after symptom onset. Despite the use of TCZ, all patients presented with torpid evolution, with a higher oxygen requirement and progressive elevation of inflammatory markers (lactate dehydrogenase (LDH), ferritin, and D-dimer).

A median 5 days after TCZ administration, anakinra was administered for 5 days. All patients continued requiring high- flow supplemental oxygen (oxygen reservoir or non-invasive ventilation) and maintained high acute-phase reactants (Fig. 1) without clinical or radiographic improvement. Serum cytokine levels were analyzed in three patients. An exaggerated inflammatory response with elevated pro-inflammatory (IL-6, IL-7, CCL2, CXCL10) and anti-inflammatory (IL-10) cytokines was observed despite the use of anakinra (Supplemental Fig. 2). Unfortunately, all patients died from severe respiratory failure a median 6 days (range 1-29 days) after anakinra was started.

All of our cases presented a hyperinflammatory state consistent with CRS/MAS induced by COVID-19 and refractory to treatment with TCZ. After the administration of anakinra, clinical, radiological, and inflammatory markers continued to worsen (Fig. 1). C-reactive protein levels decreased prior to the use of anakinra as an effect of the treatment with TCZ.

Analyzing the levels of various cytokines in these patients, we observed an undetectable serum IL1B level, though IL1Ra increased after anakinra infusion (Supplemental Fig. 2). The fact that IL1B values were not elevated in the peripheral blood does not exclude the possibility that they were increased locally. Notably, we not only observed little change in the levels of CCL2 and CXCL10 (indicators of MAS) after administration of the IL1Ra, but we observed an increase in IL-10, the most immunosuppressive cytokine. This may lead to a different understanding of the pathophysiology of the disease and a different therapeutic approach. Similarly, there is concern that suppressing the innate and adaptive immune systems to address increased cytokine concentrations, such as elevated IL-6, could enable unfettered viral replication, suppress adaptive immunity, and delay recovery processes $[9$, 10]. No clinical benefits were shown with the administration of anakinra in this group of hematological patients. The use of complementary drugs acting against viral replication and at different sites in the MAS inflammatory 
cascade could demonstrate a clinical benefit in hematological patients with COVID-19. Furthermore, taking into account the possibility of an increase in the viral replication of SARS-CoV-2 due to the immunosuppression generated by the treatment, the ideal moment to stop the inflammatory response remains to be elucidated.

Although our study has limitations due to the small sample size, our data highlight that the use of off-label drugs would best be utilized in the context of a randomized clinical trial of the timing of anakinra administration in the disease course and indications based on clinical parameters and biomarkers. Based on our experience, IL1R-blockade should not be recommended in patients with severe SARS-CoV-2 infection and hematological malignancies.

Acknowledgments CV, MP and JML made contributions to the acquisition, analysis and interpretation of data; all the authors made de critical revision of the paper and approved the submitted and final versions.

Funding information This work is partially supported by CRIS foundation for cancer research and COV20/00181 grant from Fondo de investigaciones sanitarias of the Spanish Health System.

\section{Compliance with ethical standards}

Conflict of interest The authors declare that they have no conflict of interest.

Ethical approval All procedures performed in studies involving human participants were in accordance with the ethical standards of the institutional and/or national research committee and with the 1964 Helsinki declaration and its later amendments or comparable ethical standards.

Informed consent Informed consent was obtained from all individual participants included in the study.

\section{References}

1. Organization WH. 2020 Novel coronavirus-China. World Health Organization cited January 20

2. Center for Systems Science and Engineering. Coronavirus COVID19 global cases.2019 (https://gisanddata.maps.arcgis.com/apps/ opsdashboard/index.html\#/bda7594740fd40299423467b48e9ecf6. opens in new tab). 2020

3. Ruan Q, Yang K, Wang W, Jiang L, Song J (2020) Clinical predictors of mortality due to COVID-19 based on an analysis of data of 150 patients from Wuhan. China. 46(5):846-848

4. Shakoory B, Carcillo JA, Chatham WW, Amdur RL, Zhao H, Dinarello CA, Cron RQ, Opal SM (2016) Interleukin-1 receptor blockade is associated with reduced mortality in sepsis patients with features of macrophage activation syndrome: reanalysis of a prior phase III trial. Crit Care Med 44:275-281

5. Moore BJB, June CH (2020) Cytokine release syndrome in severe COVID-19. Science. 368:473-474

6. Cavalli G, De Luca G, Campochiario C et al (2020) Interleukin-1 blockade with high-dose anakinra in patients with COVID-19, acute respiratory distress syndrome, and hyperinflammation: a retrospective cohort study (published online ahead of print 7 May 2020). Lancet Rheumatol. https://doi.org/10.1016/S26659913(20)30127-2

7. $\mathrm{Xu}$ X, Han M, Li T et al Effective treatment of severe COVID-19 patients with tocilizumab (published online ahead of print 29 April 2020). Proc Natl Acad Sci U S A. https://doi.org/10.12074/202003. 00026

8. Dinarello CA (2018) Overview of the IL-1 family in innate inflammation and acquired immunity. Immunol Rev 281(1):8-27

9. Schulert GS, Grom AA (2015) Pathogenesis of macrophage activation syndrome and potential for cytokine directed therapies. Annu Rev Med 66:145-159

10. Remy KE, Brakenridge SC, Francois B et al Immunotherapies for COVID-19: lessons learned from sepsis (published online ahead of print 28 April 2020). Lancet. https://doi.org/10.1016/S22132600(20)30217-4

Publisher's note Springer Nature remains neutral with regard to jurisdictional claims in published maps and institutional affiliations. 